\title{
Assessment of left ventricular twist mechanics by speckle tracking echocardiography reveals association between $L V$ twist and myocardial fibrosis in patients with hypertrophic cardiomyopathy
}

\author{
Hong-Ju Zhang $\cdot$ Hao Wang $\cdot$ Tao Sun $\cdot$ \\ Min-Jie Lu • Nan Xu • Wei-Chun Wu • \\ Xin Sun • Wu-Gang Wang • Qiong-Wen Lin \\ Received: 31 March 2014/ Accepted: 29 July 2014/Published online: 9 August 2014 \\ (c) The Author(s) 2014. This article is published with open access at Springerlink.com
}

\begin{abstract}
We aimed to investigate whether left ventricular (LV) twist analysis can detect the extent of myocardial fibrosis in patients with hypertrophic cardiomyopathy (HCM). This prospective case-control study recruited 81 consecutive patients with HCM examined between January 2012 and April 2013. Data of 76 patients were analyzed after excluding 5 patients whose echocardiographic images were of poor quality. Healthy volunteers $(n=46)$ served as controls. Both groups underwent comprehensive echocardiographic examination (i.e., Bas-Rotation, AP-Rotation, LVEF, LADs, IVST, LAVi, E/Em, LVMI, advanced LV-twist analysis by speckle tracking echocardiography) and magnetic resonance imaging. Between-group differences were analyzed by independent $t$ test; logistic regression analysis was performed to identify effect factors. No significant differences were found between baseline characteristics of HCM and control groups (all $p>0.05)$. HCM patients had significantly higher BasRotation, AP-Rotation, LV Twist, LVEF, LADs, IVST,
\end{abstract}

Hong-Ju Zhang and Tao Sun are co-first Authors

Electronic supplementary material The online version of this article (doi:10.1007/s10554-014-0509-6) contains supplementary material, which is available to authorized users.

H.-J. Zhang · H. Wang (ه) - M.-J. Lu · N. Xu · W.-C. Wu

X. Sun · W.-G. Wang · Q.-W. Lin

Department of Ultrasound, State Key Laboratory of

Cardiovascular Disease, Fuwai Hospital, National Center for Cardiovascular Diseases, Chinese Academy of Medical Sciences and Peking Union Medical College, No. 167, Bei Lishi Road,

Xicheng District, Beijing 100037, People's Republic of China

e-mail: hal6112@hotmail.com

T. Sun

Division of Cardiology, Beijing Anzhen Hospital, Capital

Medical University, Beijing 100029, People's Republic of China
LAVi, E/Em and LVMI than controls (all $p<0.0001$ ) and significantly lower LVDd and E/A (both $p<0.001$ ). BasRotation, AP-Rotation, LV-Twist, LADs, IVST, LAVi, E/Em and LVMI were significantly higher in HCM patients with fibrosis than in those without fibrosis $(p<0.001)$, but no significant differences in other echocardiographic parameters were found between those with and without fibrosis. Age, Bas-Rotation, AP-Rotation, LV twist, LADs, IVST, LAVi, E/A, E/Em, and LVMI were significant effect factors for fibrosis. AUROC analysis showed that LV twist had high discriminatory power to detect extent of myocardial fibrosis (AUC 0.996, $95 \%$ CI 0.989-1.004, $p<0.001)$. Left ventricular twist mechanics are associated with the extent of myocardial fibrosis. LV-twist assessment by STE may be clinically useful.

Keywords Hypertrophic cardiomyopathy - Cardiac magnetic resonance Speckle tracking echocardiography · Myocardial fibrosis

\section{Introduction}

Myocardial fibrosis is a pathological entity associated with extracellular matrix remodeling, which may lead to increased myocardial stiffness and left ventricular systolic and diastolic dysfunction [1, 2]. Late gadolinium enhancement (LGE) by cardiovascular magnetic resonance imaging (CMRI) is frequently observed in patients with hypertrophic cardiomyopathy (HCM) and allows in vivo quantification of myocardial fibrosis [3, 4]. Previous studies in large HCM cohorts have noted that delayed enhancement by CMRI such as LGE is associated with left ventricular (LV) systolic and diastolic dysfunction, including increased risk of non-sustained ventricular 
tachycardia (NSVD) that may lead to adverse cardiac events such as sudden cardiac death, fatal arrhythmia, or worsening heart failure in HCM patients [3, 4]. Thus, it becomes important to identify HCM patients at higher risk for ventricular arrhythmias and sudden cardiac death [1]. However, although contrast-enhanced cardiovascular MRI like LGE is able to identify the underlying abnormal myocardial substrate that comprises fibrosis [3], the clinical significance of these findings is still not well understood [4]. Besides the prognostic value of delayed contrast enhancement in CMRI [5], evaluation of other parameters may be needed to accurately evaluate risk in HCM patients.

Speckle tracking echocardiography (STE), which is based on tracking and measurement of tissue displacement, has the potential to accurately and reliably assess myocardial mechanics, providing a relatively simple, noninvasive approach to the study of LV rotation and twist [6-9]. LV twist represents the mean longitudinal gradient of the difference in clockwise and counter-clockwise rotation of the left ventricular apex and base, a phenomenon that links systolic contraction with diastolic relaxation and plays a major role in cardiac physiology [10]. Choudhury et al. [11] reported that regional myocardial strain and wall thickening were affected by regional myocardial fibrosis in patients with HCM. LV rotation occurs when myocardial fibers contract and, as such, impaired LV twist mechanics may be the basis of LV dysfunction in the presence of acute increase in afterload [12]. However, the association between LV twist and the extent of myocardial fibrosis is unknown, and the clinical significance of LV twist mechanics remains unclear in HCM patients. To the best of our knowledge, studies examining the relationship between LV twist mechanics and the extent of myocardial fibrosis have not been published. We hypothesized that the presence of greater myocardial twist may be associated with a greater degree of myocardial fibrosis in patients with HCM and that LV twist mechanics assessment may help differentiate the presence of fibrosis. We expected that the relationship between LV twist and myocardial fibrosis could be confirmed by using STE in conjunction with other echocardiographic parameters and MRI imaging. Therefore, the aim of this study was to investigate whether assessment of LV twist mechanics by STE is able to detect the extent of myocardial fibrosis and serve as a novel prognostic parameter in HCM patients.

\section{Methods}

\section{Patients}

This prospective case-control study recruited a total of 81 consecutive patients with HCM who were examined in our department between January 2012 and April 2013. The diagnosis of HCM was made by echocardiography based on the criteria of the World Health Organization/International Society and Federation of Cardiology. All patients had also undergone MRI examination. Patients' medical records were thoroughly reviewed to ensure that there was no history of hypertension, and no patient presented with hypertension during the study period. Patients were excluded if they had poor resolution of echocardiographic images; obstructive HCM (LV outflow tract gradients $>30 \mathrm{mmHg}$ under basal conditions or after Valsalva maneuver); left ventricular ejection fraction (LVEF) $<50 \%$ as assessed by echocardiography; cardiac muscle disease secondary to any known systemic condition; atrial fibrillation, implantation of a pacemaker or defibrillator; significant valvular heart disease; or known coronary artery disease (lesions $>50 \%$ on angiogram). Finally, after five patients were excluded because their echocardiographic images were of poor quality and not suitable for the present study, the data from 76 patients were retained for subsequent analysis.

Patients were classified as having asymmetric septal hypertrophy if wall thickness in the basal or mid anterior/ inferior septum was $>1.3 \mathrm{~cm}$ and septal/lateral wall thickness ratio was $>1.3$. Patients were classified as having a septal bulge if the proximal anterior/inferior septum was $>1.3 \mathrm{~cm}$ and the mid or apical anterior/inferior septum was $<1.3 \mathrm{~cm}$. Apical hypertrophy was diagnosed if the ratio of maximal segmental wall thickness of the apex and the rest of the ventricle was $>1.5$. All patients were in sinus rhythm.

All drugs were discontinued at least $24 \mathrm{~h}$ before echocardiography and MRI evaluation, as previously recommended [13]. Patient history of syncope (transient and complete loss of consciousness) and family history of sudden cardiac death in a first-degree relative were recorded.

For the purpose of analysis, the HCM group $(n=76)$ was further divided (after imaging and echocardiographic examinations described below) into a fibrosis group and a non-fibrosis group.

In addition, a group of 46 healthy volunteers who underwent advanced LV-twist analysis by echocardiography served as controls. These subjects had no documented history of cardiovascular disease, including hypertension or HCM. These subjects did not undergo MRI evaluation.

\section{Ethical considerations}

This study was reviewed and approved by the hospital's Institutional Review Board. All included patients and control subjects provided signed informed consent.

MRI examination and image analysis

Magnetic resonance imaging examination was performed within 2 weeks of echocardiographic examination. MRI was performed using a 1.5-T Sonata scanner (Siemens 
Corporation, Erlangen, Germany). Electro cardio-gating breath-holding sequential scanning was carried out with the patient in the dorsal position. Axial and sagittal single-shot turbo spin echo imaging was carried out on the basis of conventional axial, sagittal and coronal phases. Cineimaging was carried out for left ventricular two-chamber and four-chamber longitudinal planes as well as LV outflow tract planes and LV shot-axis view for 6-8 layers, and the scanning sequence was retrospective ECG gating true fast imaging, as described previously [14]. The left ventricular mass at end-diastole was semi-automatically calculated on the basis of Simpson's rule, using a work station and manual revision by visual assessment. The left ventricular mass was normalized to body surface area. Images for LGE diagnostics were acquired $10 \mathrm{~min}$ after the injection of gadopentetate dimeglumine $0.2 \mathrm{mmol} / \mathrm{kg}$ (Magnevist; Schering Corporation, Berlin, Germany) with breath-hold segmented inversion-recovery sequences acquired in the same view. Myocardial perfusion and delayed scanning were analyzed according to the 17-segment method for the left ventricle recommended by the American Heart Association (AHA) [15]. The presence of late enhancement within each segment was assessed semiquantitatively using a 17 -segment model of the LV [16]. LGE of the LV was considered present if the signal intensity of hyperenhanced myocardium was $>5$ standard deviations above the mean signal intensity of vital myocardium. MRI analysis was completely blinded from the echocardiographic and clinical analysis.

\section{Echocardiography examination}

Echocardiography was carried out using the Phillips iE33 Color Doppler Ultrasound System with probe frequency $3.5 \mathrm{MHz}$ (Philips Healthcare, Andover, MA, USA). The Qlab multiparameter analysis workstation (Phillips) was equipped with STE analysis software. The second-harmonic B-mode images of apical (4-chamber, 2-chamber) and short-axis (at the mitral valve and apical level) views were obtained. The frame rate was more than 50 frames/s. The LV endocardial border was manually traced at the endsystolic frame and the speckle tracking region of interest was automatically selected. The width of the region of interest was adjusted as necessary to accommodate the total thickness of the LV wall [17]. The computer automatically tracked stable objects in each frame using the sum of absolute differences algorithm. After these steps, the work station computed and generated strain curves, as previously described [18].

The peak values of basal rotation were defined as the maximum negative values of the curves from the short-axis view at the mitral valve level. The peak values of apical rotation were defined as the maximum positive values of the curves from the short-axis view at the apical level [19]. Every view considered was divided into six segments, giving six different values from which mean values were determined. LV twist was defined as the difference between the mean values of the peak rotation at the apical and mitral valve level (twist $=$ mean peak apical rotation - mean peak basal rotation) [10].

Standard two-dimensional measurements were obtained using standard methods. LVEF was calculated using the two-dimensional Simpson's biplane method. LV mass was assessed with the two-dimensional area-length method and the left ventricular mass was normalized to body surface area, as previously described [20]. Left ventricular diameter in end-diastolic (LVDd), inter-ventricular septal thickness (IVST), left ventricular posterior wall thickness (LVPWT), and relative wall thickness (RWT) were measured according to criteria of the American Society of Echocardiography. Left atrium end-systolic dimensions (LADs) were measured at end-systolic, and LA volume was calculated using the prolate ellipsoid model. The LA volume index (LAVi) was calculated as LA volume divided by body surface area, reported as milliliters per square meter. The following variables were measured at the tips of the mitral valve leaflets from the apical 4-chamber view using pulsedwave Doppler: peak early (E) and late (A) diastolic velocities, and E/A ratio were measured. Finally, by pulsed tissue Doppler, peak early diastolic velocity on the septal part of the mitral annulus was measured (Em) and $\mathrm{E} / \mathrm{Em}$ ratio was calculated as previously described [20].

Statistical analysis

Continuous variables were presented as mean and standard deviation, with independent $t$ test used to compare differences between groups. Categorical variables were presented as counts and percentages, with Fisher's exact tests used to compare differences between groups. Logistic regression was performed to detect which factor provides the strongest influence among myocardial fibrosis groups. Factors that were significant in univariate logistic regression were included in multivariate logistic regression, with stepwise model selection used to select the best factor to detect myocardial fibrosis. The receiver operating characteristic (ROC) curve was used to examine the factor performance in fibrosis and non-fibrosis groups. The cut-off value of factors that may distinguish myocardial fibrosis or not were selected using Youden index, which was defined as sensitivity + specificity -1 . Area under ROC curve with null hypothesis: $\mathrm{AUC}=0.5$ was performed using the Wilcoxon rank sum test. All statistical analyses were performed with SPSS software version 17 (SPSS Inc, Chicago, IL, USA), and two-tailed $p<0.05$ indicated statistical significance. 
Table 1 Baseline characteristics of patients in HCM and control groups

\begin{tabular}{lllr}
\hline & $\begin{array}{l}\text { HCM group } \\
(\mathrm{N}=76)\end{array}$ & $\begin{array}{l}\text { Control group } \\
(\mathrm{N}=46)\end{array}$ & $p$ value \\
\hline Age(years) & $47.1 \pm 12.6$ & $44.3 \pm 6.9$ & 0.116 \\
Gender & $50(65.8 \%)$ & $32(69.6 \%)$ & 0.667 \\
$\quad$ Male & $26(34.2 \%)$ & $14(30.4 \%)$ & \\
Female & $70.5 \pm 9.4$ & $70.4 \pm 2.5$ & 0.982 \\
Heart rate (beats/min) & $1.7 \pm 0.2$ & $1.7 \pm 0.1$ & 0.707 \\
BSA $\left(\mathrm{m}^{2}\right)$ & $24.1 \pm 2.0$ & $23.5 \pm 1.5$ & 0.101 \\
BMI $\left(\mathrm{kg} / \mathrm{m}^{2}\right)$ & $124.3 \pm 6$ & $119.5 \pm 7.1$ & $<0.001$ \\
SBP $(\mathrm{mmHg})$ & $81.8 \pm 5.8$ & $78 \pm 5.1$ & $<0.001$ \\
DBP $(\mathrm{mmHg})$ & $20(26.3 \%)$ & $0(0 \%)$ & $<0.001$ \\
Family history of HCM & $10(13.2 \%)$ & $0(0 \%)$ & $<0.001$ \\
Unexplained syncope & & & \\
\hline
\end{tabular}

\section{Results}

Comparison of baseline characteristics in $\mathrm{HCM}$ and control groups

Patients' baseline characteristics in HCM and control groups are presented in Table 1. Among 122 subjects enrolled in this study, 76 were HCM patients and 46 were healthy subjects, including 82 males and 40 females with a mean age of 46.1 years. Mean heart rate was 70.5 beats/ min, mean BSA was $1.7 \mathrm{~m}^{2}$, mean BMI was $23.9 \mathrm{~kg} / \mathrm{m}^{2}$, mean SBP was $122.5 \mathrm{mmHg}$ andmean DBP was $80.4 \mathrm{mmHg}$. In the HCM group, $26.3 \%$ had a history of HCM and $13.2 \%$ had unexplained syncope.

No significant differences were found between the HCM and control group in age, gender, heart rate, BSA and BMI $(p>0.05)$. The means of SBP and DBP were significantly higher in the HCM group than in the control group (SBP: 124.3 vs. $119.5, p<0.001$; DBP: 81.8 vs. $78, p<0.001$ ). The HCM group had more patients with a history of HCM and unexplained syncope than the control group (history of HCM: 20 vs. $0, p<0.001$; unexplained syncope: 10 vs. 0 , $p<0.001$ ) (Table 1).

Comparison of echocardiography parameters in HCM and control groups

Comparisons of echocardiography parameters between HCM and control groups are presented in Table 2. Among 122 patients, the mean Bas-Rotation was $7.4^{\circ}$, mean APRotation was $9.9^{\circ}$, mean LV-Twist was $17.3^{\circ}$, mean LVEF was $69.2 \%$, mean LADs was $37.4 \mathrm{~mm}$, mean LVDd was $42.3 \mathrm{~mm}$, mean IVST was $16.3 \mathrm{~mm}$, mean LAVi was $43.5 \mathrm{ml} / \mathrm{m}^{2}$, mean E/A was 1.1 , mean E/Em was 12.3 and
Table 2 Comparison of echocardiographic parameters in HCM and control groups

\begin{tabular}{lccr}
\hline & HCM $(\mathrm{N}=76)$ & Control $(\mathrm{N}=46)$ & $p$ value \\
\hline Bas-Rotation $\left(^{\circ}\right)$ & $8.6 \pm 1.8$ & $5.5 \pm 0.7$ & $<0.001$ \\
AP-Rotation $\left(^{\circ}\right)$ & $11.2 \pm 2.4$ & $7.8 \pm 0.6$ & $<0.001$ \\
LV-Twist $\left(^{\circ}\right)$ & $19.8 \pm 4.0$ & $13.2 \pm 0.9$ & $<0.001$ \\
LVEF $(\%)$ & $70.4 \pm 6.0$ & $67.3 \pm 3.8$ & 0.001 \\
LADs $(\mathrm{mm})$ & $40.6 \pm 3.1$ & $32.1 \pm 2.0$ & $<0.001$ \\
LVDd $(\mathrm{mm})$ & $41.0 \pm 2.1$ & $44.5 \pm 1.5$ & $<0.001$ \\
LAVi $\left(\mathrm{ml} / \mathrm{m}^{2}\right)$ & $51.3 \pm 5.4$ & $30.7 \pm 2.9$ & $<0.001$ \\
IVST $(\mathrm{mm})$ & $20.7 \pm 3.0$ & $9.0 \pm 0.6$ & $<0.001$ \\
LVPWT $(\mathrm{mm})$ & $10.9 \pm 0.8$ & $8.8 \pm 0.4$ & $<0.001$ \\
RWT & $0.5 \pm 0.04$ & $0.3 \pm 0.01$ & $<0.001$ \\
E/A & $1.0 \pm 0.3$ & $1.3 \pm 0.1$ & $<0.001$ \\
E/Em & $14.6 \pm 3.2$ & $8.5 \pm 1.1$ & $<0.001$ \\
LVMI $\left(\mathrm{g} / \mathrm{m}^{2}\right)$ & $119.1 \pm 22.5$ & $68.9 \pm 7.6$ & $<0.001$ \\
GLS & $-14.2 \pm 1.8$ & $-18.0 \pm 0.4$ & $<0.001$ \\
Untwisting velocity & $-10.5 \pm 1.1$ & $-13.1 \pm 0.4$ & $<0.001$ \\
\hline
\end{tabular}

mean LVMI was $100.2 \mathrm{~g} / \mathrm{m}^{2}$. The HCM group had significantly higher values for Bas-Rotation, AP-Rotation, LV-Twist, LVEF, LADs, LAVi, IVST, LVPWT, RWT, $\mathrm{E} / \mathrm{Em}$ and LVMI than the control group (all $p<0.0001$ ). LVDd and E/A were significantly lower in the HCM group than in the control group (both $p<0.001$ ) (Table 2). Representative examples are shown in Fig. 1.

Distribution of characteristics in HCM patients with and without fibrosis

The distribution of characteristics in HCM patients with and without fibrosis is presented in Table 3. In the $76 \mathrm{HCM}$ patients, means for Bas-Rotation, AP-Rotation, LV-Twist, LADs, LAVi, IVST, LVPWT, RWT, E/Em, and LVMI were all significantly higher in the fibrosis group than in the non-fibrosis group $(p<0.001)$. Means of age and E/A were significantly lower in the fibrosis group compared with non-fibrosis group (age: 44.6 vs. 51.7 years, $p=0.007$; E/A: 1.0 vs. $1.2, p=0.001$ ). No significant differences were found between non-fibrosis vs. fibrosis groups in heart rate, BSA, BMI, SBP, DBP and LVEF $(p>0.05)$. Representative examples are shown in Fig. 2.

In addition, LV-twist showed a significant, positive and linear correlation with IVST, LVPWT, RWT, LAVi and $\mathrm{E} / \mathrm{Em}$ in $\mathrm{HCM}$ patients with fibrosis. The greater the increase in LV-twist, the greater were IVST, LVPWT, RWT, LAVi and E/Em. No significant linear correlations were found between LV-twist and $\mathrm{E} / \mathrm{A}$, untwisting velocity (Supplemental Table 2). 
Fig. 1 Two-dimensional speckle tracking images in the short-axis view at the mitral valve and apical level are shown. Apical rotation, basal rotation, and LV twist in $\mathrm{HCM}$ patients is significantly higher than in controls. $H C M$ hypertrophic cardiomyopathy

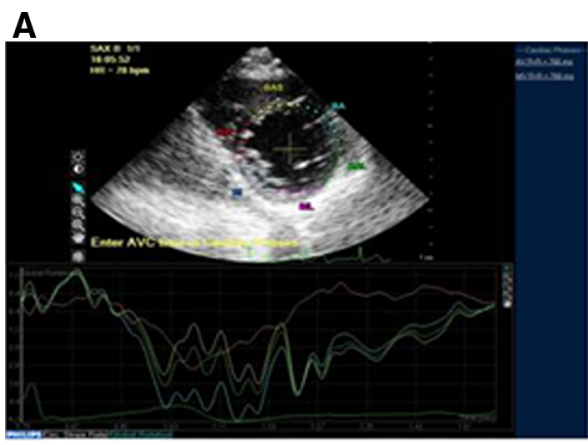

basal rotation of Controls

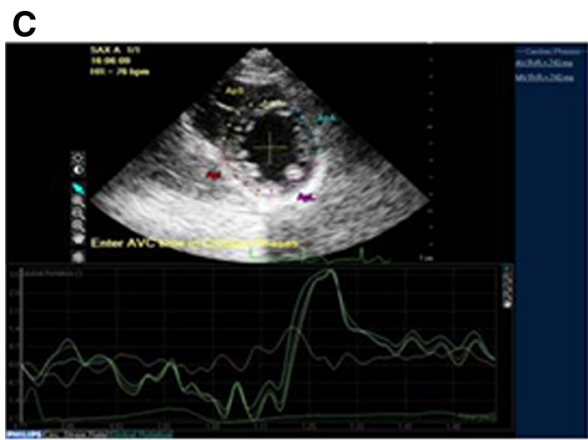

apical rotation of Controls

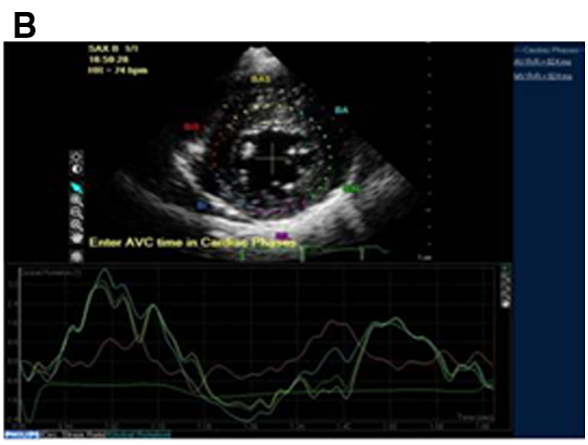

basal rotation of HCM

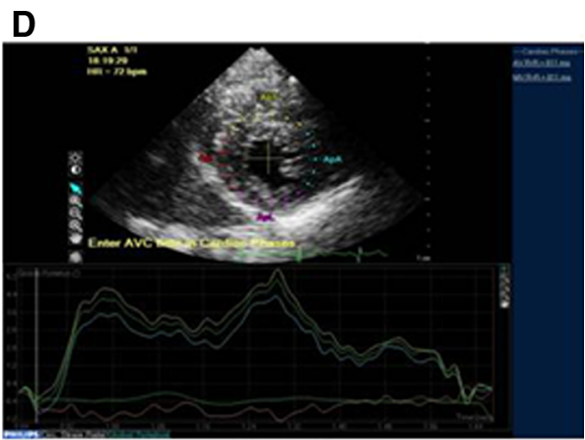

apical rotation of HCM
Table 3 Distribution of characteristics in HCM patients with and without fibrosis

\begin{tabular}{lccc}
\hline & $\begin{array}{c}\text { Non-fibrosis } \\
(\mathrm{N}=27)\end{array}$ & $\begin{array}{l}\text { Fibrosis group } \\
(\mathrm{N}=49)\end{array}$ & $p$ value \\
\hline Age (years) & $51.7 \pm 8.5$ & $44.6 \pm 13.8$ & $0.007^{*}$ \\
Heart rate (beats/min) & $70.7 \pm 10.6$ & $70.4 \pm 8.9$ & 0.889 \\
BSA $\left(\mathrm{m}^{2}\right)$ & $1.7 \pm 0.2$ & $1.8 \pm 0.1$ & 0.129 \\
BMI $\left(\mathrm{kg} / \mathrm{m}^{2}\right)$ & $24.2 \pm 1.6$ & $24.0 \pm 2.3$ & 0.693 \\
SBP $(\mathrm{mmHg})$ & $123.7 \pm 5.9$ & $124.6 \pm 6.1$ & 0.524 \\
DBP $(\mathrm{mmHg})$ & $81.4 \pm 5.5$ & $82.1 \pm 5.9$ & 0.628 \\
Bas-Rotation $\left({ }^{\circ}\right)$ & $6.7 \pm 0.9$ & $9.6 \pm 1.3$ & $<0.001^{*}$ \\
AP-Rotation $\left({ }^{\circ}\right)$ & $8.3 \pm 0.9$ & $12.7 \pm 1.2$ & $<0.001^{*}$ \\
LV-Twist $\left(^{\circ}\right)$ & $15.0 \pm 1.1$ & $22.4 \pm 2.1$ & $<0.001^{*}$ \\
LVEF $(\%)$ & $70.7 \pm 3.7$ & $70.2 \pm 6.9$ & 0.698 \\
LADs $(\mathrm{mm})$ & $38.7 \pm 1.1$ & $41.7 \pm 3.3$ & $<0.001^{*}$ \\
LVDd $(\mathrm{mm})$ & $41.6 \pm 1.9$ & $40.7 \pm 2.1$ & 0.052 \\
LAVi $\left(\mathrm{ml} / \mathrm{m}^{2}\right)$ & $45.1 \pm 2.4$ & $54.7 \pm 2.9$ & $<0.001^{*}$ \\
IVST $(\mathrm{mm})$ & $18.3 \pm 2.2$ & $22.1 \pm 2.4$ & $<0.001^{*}$ \\
LVPWT $(\mathrm{mm})$ & $10.7 \pm 0.7$ & $11.1 \pm 0.8$ & $0.02^{*}$ \\
RWT & $0.5 \pm 0.04$ & $0.5 \pm 0.02$ & $<0.001^{*}$ \\
E/A & $1.2 \pm 0.2$ & $1.0 \pm 0.2$ & $0.001^{*}$ \\
E/Em & $11.1 \pm 1.6$ & $16.6 \pm 1.9$ & $<0.001^{*}$ \\
LVMI (g/m $\left.{ }^{2}\right)$ & $98.3 \pm 10.5$ & $130.6 \pm 18.8$ & $<0.001^{*}$ \\
GLS & $-16.4 \pm 0.9$ & $-13.0 \pm 0.6$ & $<0.001^{*}$ \\
Untwisting velocity & $-11.4 \pm 1.1$ & $-10.0 \pm 0.8$ & $<0.001^{*}$ \\
\hline
\end{tabular}

* Significant differences between non-fibrosis versus fibrosis groups
Univariate and multivariate logistic regression analysis of effect factors for fibrosis in HCM patients

Univariate logistic regression revealed that the significant effect factors for fibrosis in HCM patients were age, BasRotation, AP-Rotation, LV-Twist, LADs, IVST, LAVi, E/A, E/Em, LVMI, LVPWT and RWT. When these variables were recruited into multivariate logistic regression, stepwise model selection revealed that LV-twist would be the best factor to detect myocardial fibrosis (Supplemental Table 1). When ROC curve analysis was used to examine the LV-Twist performance in HCM patients with and without myocardial fibrosis, the AUC showed high discriminatory power for LV-twist to distinguish between myocardial fibrosis or no fibrosis (AUC 0.99, $95 \%$ CI $0.99-1.0, p<0.001$, Fig. 3); with an optimal cut-off value of 18.5 for LV-Twist as the lower limit at which to detect patients with fibrosis. Youden index determined sensitivity and specificity to be 98 and $100 \%$, respectively. The AUC also showed high discriminatory power for GLS and E/Em to distinguish between myocardial fibrosis or no fibrosis (GLS: AUC 1.0, $95 \%$ CI 1.0-1.0, $p<0.001$; E/Em: AUC $0.99,95 \%$ CI $0.99-1.0, p<0.001$ ) (Fig. 3); with an optimal cut-off value of -14.7 for GLS and 13.4 for E/Em as the lower limit at which to detect patients with fibrosis. Youden index determined sensitivity and specificity to be 100 and $100 \%$ for GLS, and 98 and $96.3 \%$ for E/Em (Table 4). 
Fig. 2 Images are shown for an HCM patient with large LGE

(a) and a patient without LGE

(b). LV twist in the patient with LGE(c) was higher than that in the patient without LGE (d). $H C M$ hypertrophic cardiomyopathy, $L G E$ late gadolinium enhancement, $L V$ left ventricular
A

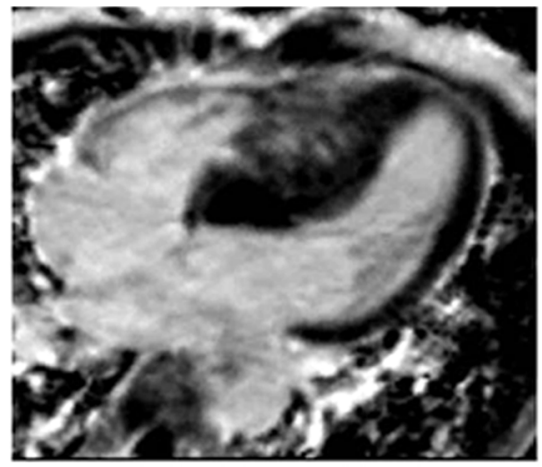

HCM patient with LGE

C

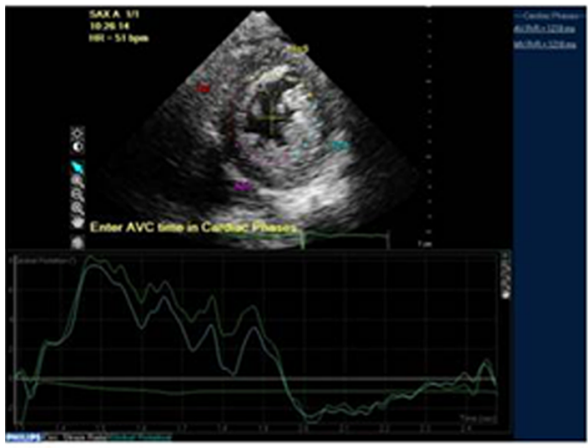

apical rotation of patient with LGE
B

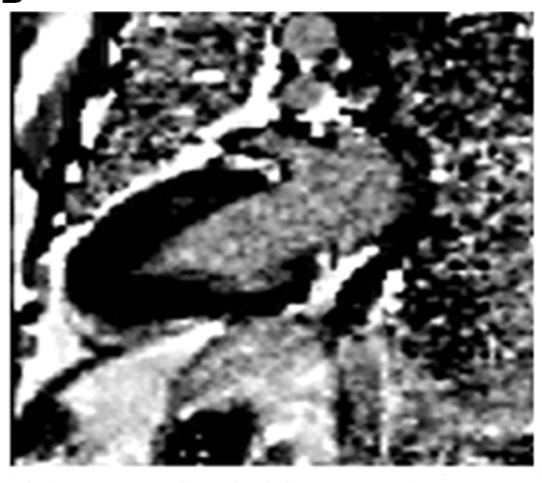

HCM patient without any LGE

D

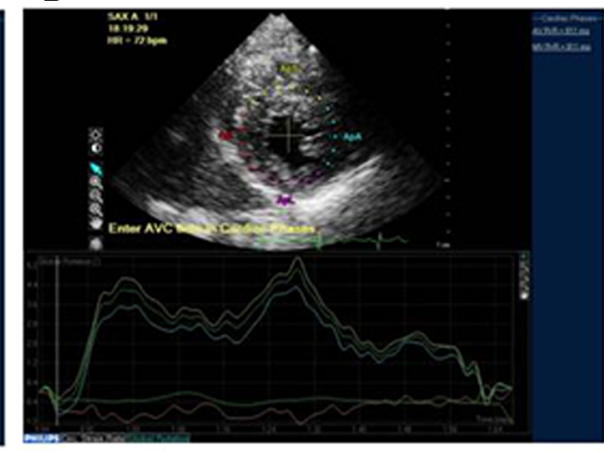

apical rotation of patient without LGE

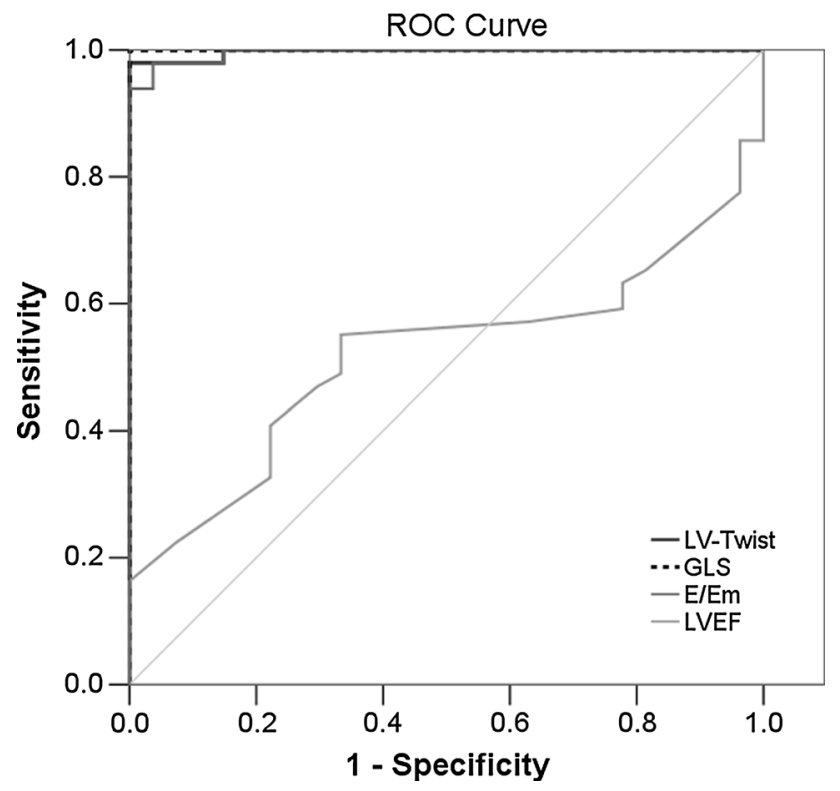

Fig. 3 The receiver operating characteristic (ROC) curves for LVtwist, GLS, E/Em and LVEF in HCM patients with and without fibrosis. GLS global 2-dimensional strain

\section{Discussion}

Results of the present study demonstrate that patients with $\mathrm{HCM}$, even in the presence of preserved EF, show impaired regional myocardial deformation with increased LV twist. LV twist is significantly associated with myocardial fibrosis segments, suggesting that LV twist may provide useful information on the presence and extent of myocardial fibrosis and cardiac events in HCM patients with normal LVEF. Differences in diagnostic parameters between HCM patients and controls.

Our results showed that the apical horizontal rotation angle, base rotation angle and left ventricular torsion angle in HCM patients were significantly higher than those in healthy controls. This might be explained by the fact that cardiac hypertrophy in HCM patients may increase the moment of epicardial fibers, resulting in dominant counterclockwise rotation of the epicardial fibers. Miyazaki et al. [21] studied HCM patients at the molecular level, showing that the differential pressure across the walls due to the decrement of light chain-related phosphorylated 
Table 4 Optimal cutoff value of LV twist by Youden index summary of AUROC curve
$G L S$ global two-dimensional strain

\begin{tabular}{llllll}
\hline & $\begin{array}{l}\text { Optimal } \\
\text { Cutoff value }\end{array}$ & $\begin{array}{l}\text { Sensitivity } \\
(95 \% \mathrm{CI})\end{array}$ & $\begin{array}{l}\text { Specificity } \\
(95 \% \mathrm{CI})\end{array}$ & $\begin{array}{l}\text { AUC } \\
(95 \% \mathrm{CI})\end{array}$ & $p$ value \\
\hline LV-twist & $\geq 18.5$ & $98 \%$ & $100 \%$ & 0.99 & $<0.001$ \\
& & $(87.8-99.9 \%)$ & $(84.5-100 \%)$ & $(0.99-1.0)$ & $<0.001$ \\
GLS & $\geq-14.7$ & $100 \%$ & $100 \%$ & $1.0(1.0-1.0)$ & \\
& & $(92.7-100 \%)$ & $(87.2-100 \%)$ & & $0.99(0.99-1.0)$ \\
E/Em & 913.4 & $(89.1-99.9 \%)$ & $(81.0-99.9 \%)$ & & $<0.001$ \\
& & $55.1 \%$ & $66.7 \%$ & $0.5(0.4-0.6)$ & 0.78 \\
LVEF & $\geq 70.5$ & $(40.2-69.3 \%)$ & $(46.0-83.5 \%)$ & & \\
\end{tabular}

myosin concentration may promote left ventricular rotation. The hypertrophic myocardium protrudes into the ventricular cavity, which reduces the left ventricular volume. Therefore, to maintain the normal ejection fraction, the ventricular contraction may increase as compensation, but the left ventricular contraction occurs by auto-rotation. Our results also showed that $\mathrm{LV}$ and EF, two parameters commonly used to evaluate the left ventricular contraction, were comparable between the HCM patients and controls. This suggests that the left ventricular contraction in HCM at the early stage is still compensatory, and traditional echocardiography usually fails to reflect early left ventricle dysfunction. However, STE may sensitively reflect the dysfunction of the left ventricle in patients with HCM at an early stage.

In another study, apical rotation, basal rotation and torsion or LV twist were increased in patients with $\mathrm{HCM}$ compared to results of these CMRI parameters in normal volunteers [22]. In that study, HCM patients examined by magnetic resonance tagging showed an increased degree of $\mathrm{LV}$ rotation. Owing to the after-load dependency of $\mathrm{LV}$ twist, this increased degree may be associated with increased mass/volume ratio, thus reduced afterload is characteristic of HCM patients. In the present study, while the difference in LVEF results between the HCM and control groups was not statistically significant, it is consistent with the results of Van Dalen et al. [23], who showed that apical rotation and twist (maximum) in HCM patients depend on patterns of LV hypertrophy. Thus, assessment of LV twist and rotation provides reliable quantitative evaluation of ventricular regional and global function and, as such, may be the more sensitive and specific indicator of subclinical myocardial dysfunction [24]. In general, patients with HCM have preserved LVEF as assessed by conventional methods. Serri et al. [25] reported that global 2D strain (GLS) was significantly reduced in HCM patients compared with normal control subjects, despite that the two groups had similar ejection fractions. Those authors concluded that STE can identify early abnormalities in HCM patients who have apparently normal LV ejection fractions. In the present study, GLS data (Table 4; Fig. 3) helps to explain that although HCM patients have normal LVEF, patients with myocardial fibrosis have lower GLS than those of healthy controls and HCM patients without myocardial fibrosis, which indicates the effectiveness of STE in predicting fibrosis and its prognostic value in HCM patients.

A review by Sengupta et al. [10] suggested that routine application of LV twist using the algorithm twist = mean peak apical rotation - mean peak basal rotation allows clinical differentiation of LV dysfunction found in day-today cardiology practice. Additionally, a recent case study by Tanaka et al. [26] concluded that 3-D speckle-tracking strain provides a reliable evaluation of and accurate information about true LV mechanics, identifying LV dyssynchrony using pyramidal 3-D data sets acquired in the same beats, which overcomes the limitations of 2D speckle-tracking radial strain and 2D tomographic imaging planes.

Differences between HCM patients

with and without myocardial fibrosis

In the present study, left ventricular myocardial fibrosis was detected in $49 \mathrm{HCM}$ patients $(65.5 \%)$, and LV twist and apical rotation were significantly higher in the fibrosis subgroup than in the non-fibrosis subgroup. This may be the result of subendocardial ischaemia with dysfunction of subendocardial fibres causing rotation of the apex in a clockwise direction [15]. Microvascular myocardial ischemia, abnormal calcium handling and mechanical dyssynchrony are other possible mechanisms that could explain abnormalities of active relaxation, while fibrosis and myocardial hypertrophy may increase passive LV stiffness [27]. In addition, results of that study showed that the amount of segmental LGE correlated strongly with segmental wall thickening measured by MRI. Similarly, the present study showed that peak LV twist in fibrosis subgroups was significantly higher than in non-fibrosis subgroups, which suggests that LV twist, as well as LV 
rotation, is also affected by the extent of myocardial fibrosis.

We also found differences between groups in the left ventricular torsion angle, which was significantly larger in the fibrosis group than in the non-fibrosis group. This may be ascribed to subendocardial myocardial hypoperfusion due to myocardial fibrosis, affecting endomyocardial function and reducing negative torsional deformation. In addition, the apical horizontal rotation angle was markedly larger than that in the non-fibrosis group, but the base rotation angle was comparable between the two groups. Thus, the overall left ventricular rotation angle in the fibrosis group increased significantly compared to that in the non-fibrosis patients. Popovic et al. [28] applied STE to analyze the relationship between the left ventricular longitudinal strain and the number of myocardial fibrotic segments. Their results showed that the left ventricular longitudinal strain was negatively associated with the number of myocardial fibrotic segments. This suggests that the larger the area of myocardial fibrosis, the smaller the left ventricular longitudinal strain. In addition, our findings also revealed that the ventricular septum in the fibrosis group was thicker than that in non-fibrosis group. It appears that the thicker the ventricular wall, the more severe is the ischemia of myocardial microcirculation; in turn, myocardial microcirculation ischemia may induce myocyte apoptosis and deposition of collagens, which facilitates myocardial fibrosis.

In the present study, the E/Em and left atrial volume index in the fibrosis group were significantly higher than those in the non-fibrosis group. This may be ascribed to the myocardial interstitial fibrosis and the subsequent increase in ventricular wall stiffness and ventricular wall tension. In addition, the reduction in myocardial relaxation capability and compliance may have resulted in restricted ventricular filling in the early diastolic phase, with an increase in left atrial pressure and subsequent elevation in left atrial volume and $\mathrm{E} / \mathrm{Em}$. Additionally, the anteroposterior diameter in the left atrial systolic phase was comparable between the fibrosis group and non-fibrosis group, but marked difference was observed in left atrial volume index. This implies that the frequently used left atrial anteroposterior diameter may not accurately reflect the actual size of the left atrium.

Results of the present study revealed no differences in LVEF between the two subgroups. However, we observed that patients with fibrosis demonstrated larger left atrial volumes, LAVi and diastolic dysfunction as measured by E/A-ratio and tissue Doppler-derived methods (i.e., E/Emratio) as early signs for LV pathology and LV diastolic dysfunction. These data agree with results of previous studies showing early onset of markers of diastolic dysfunction in HCM patients [21, 29]. As stated above, several other mechanisms are potential causes of this functional impairment, including microvascular myocardial ischemia, abnormal calcium handling and mechanical dyssynchrony [27]. Recently, Nagakura et al. [30] evaluated LV dyssynchrony in HCM patients, finding that although LVH is not always associated with LV dyssynchrony, greater reductions in regional strain and severe dyssynchrony may be responsible in part for the adverse outcomes frequently occurring in HCM. Findings from that study showed that even when global LV systolic function is preserved, LV dyssynchrony is characteristic in HCM; genesis is typically at the apex and is believed to be due to heterogeneous myocardial wall thickening and relaxation. In an earlier study [31], application of STE revealed that deterioration of LV strain and twist mechanics may be due to a high LV outflow gradient (LVOT), since LVOT peak velocity was associated with LV mid-rotation and LV untwist in HCM patients compared to normal controls. Such evidence suggests that myocardial fibrosis appears to contribute significantly to the progressive deterioration of LV diastolic function.

Association between LV twist and the extent of myocardial fibrosis in HCM

In the present study, the left ventricular torsion angle was closely related to the number of myocardial fibrotic segments, suggesting that LV twist analysis has good sensitivity and specificity in reflecting the severity of myocardial fibrosis in HCM patients. The area under the ROC curve was 0.814 using LV twist to detect myocardial fibrosis (95\% CI $0.625-0.890$ ), which indicates that LV twist can sensitively and specifically reflect the degree of myocardial fibrosis in HCM patients. Our results are consistent with those of Saito et al. [32], who demonstrated that $2 \mathrm{D}$ global strain could identify subclinical global systolic dysfunction even though LV chamber function was normal in HCM subjects. Those authors concluded that global 2D strain was an important parameter of myocardial fibrosis in HCM patients. The present study also showed that the cutoffpoint for predicting myocardial fibrosis using LV twist was $18.22^{\circ}$, showing it to be a convenient and rapid quantitative parameter for clinical treatment and prognosis. Essentially, the larger the left ventricular torsion angle, the more severe is the myocardial fibrosis in HCM patients.

\section{Limitations}

The present study has several limitations. First, the number of patients in the study was small, which results in relatively weak statistical power. Secondly, LV twist measured by 2D speckle tracking was not actually global twist because LV twist was measured from only three short-axis views (at the mitral valve and apical level). Since the distribution of LV 
twist and hypertrophy in the left ventricular is not uniform in $\mathrm{HCM}$, it is possible that $\mathrm{LV}$ twist did not completely reflect the extent of LV myocardial fibrosis. The further development of three-dimensional (3D) speckle tracking might resolve this issue, as suggested by other authors [33]. Thirdly, the acquisition of orbicular LV short-axis images from base to apex is often technically difficult and may be settled by continued advances in 3D speckle tracking as previously suggested [34]. Fourthly, all the systolic parameters (apical rotation, basal rotation, LV twist) were heart-rate-independent because we measured peak values and did not measure timing. Finally, the ROI width at the hypertrophic segment might not cover the entire wall, because we adjusted the ROI to the thinner side of the myocardial wall. However, in the present study, LV twist did not depend on the LGE location in the myocardial wall. The development of software that can precisely trace the epicardial border might resolve this issue [33]. More clinical studies with larger patient populations are needed to confirm the validity of LV twist as a marker for myocardial fibrosis and as a predictor of major cardiac events. Future studies with larger sample-size will include patient follow-up to further confirm the relationship between left ventricular torsion angle and the severity of myocardial fibrosis. We would aim to elucidate whether the left ventricular torsion angle may be used to evaluate myocardial fibrosis or is independent of myocardial fibrosis, and whether it may predict prognosis for $\mathrm{HCM}$ patients. If so, LV twist analysis may serve as a simple, convenient indicator for clinical risk stratification and evaluation of therapeutic efficacy.

\section{Conclusions}

In conclusion, LV twist and rotation in patients with HCM can be accurately measured using STE and results may be relevant to making clinical decisions. LV twist evaluation may provide useful information on myocardial fibrosis and cardiac events in HCM patients with normal chamber function. This clinically promising parameter may be useful for risk stratification in patients with HCM.

\section{Conflict of interest None.}

Open Access This article is distributed under the terms of the Creative Commons Attribution License which permits any use, distribution, and reproduction in any medium, provided the original author(s) and the source are credited.

\section{References}

1. Leonardi S, Raineri C, De Ferrari GM, Ghio S, Scelsi L, Pasotti M, Tagliani M, Valentini A, Dore R, Raisaro A, Arbustini E (2009) Usefulness of cardiac magnetic resonance in assessing the risk of ventricular arrhythmias and sudden death in patients with hypertrophic cardiomyopathy. Eur Heart J 30:2003-2010

2. Green JJ, Berger JS, Kramer CM, Salerno M (2012) Prognostic value of late gadolinium enhancement in clinical outcomes for hypertrophic cardiomyopathy. JACC Cardiovasc Imaging 5:370-377

3. Salerno M, Kramer CM (2010) Prognosis in hypertrophic cardiomyopathy with contrast-enhanced cardiac magnetic resonance: the future looks bright. J Am Coll Cardiol 56:888-889

4. Adabag AS, Maron BJ, Appelbaum E, Harrigan CJ, Buros JL, Gibson CM, Lesser JR, Hanna CA, Udelson JE, Manning WJ, Maron MS (2008) Occurrence and frequency of arrhythmias in hypertrophic cardiomyopathy in relation to delayed enhancement on cardiovascular magnetic resonance. J Am Coll Cardiol 51:1369-1374

5. Kramer CM (2006) The expanding prognostic role of gadolinium enhanced cardiac magnetic resonance. JACC 48:1986-1987

6. Faber L, Prinz C, Welge D, Hering D, Butz T, Oldenburg O, Bogunovic N, Horstkotte D (2011) Peak systolic longitudinal strain of the lateral left ventricular wall improves after septal ablation for symptomatic hypertrophic obstructive cardiomyopathy: a follow-up study using speckle tracking echocardiography. Int $\mathrm{J}$ Cardiovasc Imaging 27:325-333

7. Helle-Valle T, Crosby J, Edvardsen T, Lyseggen E, Amundsen BH, Smith HJ, Rosen BD, Lima JA, Torp H, Ihlen H, Smiseth OA (2005) New noninvasive method for assessment of left ventricular rotation: speckle tracking echocardiography. Circulation 112:3149-3156

8. Chetboul V, Serres F, Gouni V, Tissler R, Pouchelon JL (2008) Noninvasive assessment of systolic left ventricular torsion by 2-dimensional speckle tracking imaging in the awake dog: repeatabiity, reproducibiity, and comparison with tissue Doppler imaging variables. J Vet Intern Med 22:242-250

9. Amundsen BH, Helle-Valle T, Edvardsen T, Torp H, Crosby J, Lyseggen E, Støylen A, Ihlen H, Lima JA, Smiseth OA, Slørdahl SA (2006) Noninvasive myocardial strain measurement by speckle tracking echocardiography validation against sonomicrometry and tagged magnetic resonance imaging. J Am Coll Cardiol 47:789-793

10. Sengupta PP, Tajik AJ, Chandrasekaran K, Khandheria BK (2008) Twist mechanics of the left ventricle: principles and application. JACC Cardiovasc Imaging 1:366-376

11. Choudhury L, Mahrholdt H, Wagner A, Choi KM, Elliott MD, Klocke FJ, Bonow RO, Judd RM, Kim RJ (2002) Myocardial scarring in asymptomatic or mildly symptomatic patients with hypertrophic cardiomyopahty. J Am Coll Cardiol 40:2156-2164

12. Weiner RB, Weyman AE, Kim JH, Wang TJ, Picard MH, Baggish AL (2008) The impact of isometric handgrip testing on left ventricular twist mechanics. J Physiol 590:5141-5150

13. Saito M, Okayama H, Yoshii T, Hiasa G, Sumimoto T, Inaba S, Nishimura K, Inoue K, Ogimoto A, Ohtsuka T, Funada J, Shigematsu Y, Higaki J (2011) The differences in left ventricular torsional behavior between patients with hypertrophic cardiomyopathy and hypertensive heart disease. Int J Cardiol 150:301-306

14. Popovic ZB, Kwon DH, Mishra M, Buakhamsri A, Greenberg NL, Thamilarasan M, Flamm SD, Thomas JD, Lever HM, Desai MY (2008) Association between regional ventricular function and myocardial fibrosis in hypertrophic cardiomyopathy assessed by speckle tracking echocardiography and delayed hyperenhancement magnetic resonance imaging. J Am Soc Echocardiogr 21:1299-1305

15. Prinz C, van Buuren F, Faber L, Bitter T, Bogunovic N, Burchert W, Horstkotte D (2012) Myocardial fibrosis is associated with biventricular dysfunction in patients with hypertrophic cardiomyopathy. Echocardiography 294:438-444 
16. Pacileo G, Baldini L, Limongelli G, Di Salvo G, Iacomino M, Capogrosso C, Rea A, D’ Andrea A, Russo MG, Calabrò R (2011) Prolonged left ventricular twist in cardiomyopathies: a potential link between systolic and diastolic dysfunction. Eur J Echocardiogr 12:841-849

17. Wang C, Deng YB, Zhu Y, Liu YN, Bi XJ (2012) Evaluation of subtle myocardial noncompaction by contrast echocardiography in patients with hypertrophic cardiomyopathy and its relationship with regional ventricular systolic dysfunction. J Ultrasound Med 31(10):1551-1557

18. Saito M, Okayama $H$, Yoshii $T$, Hiasa $G$, Sumimoto $T$, Inaba $S$, Nishimura K, Inoue K, Ogimoto A, Shigematsu Y, Funada J, Hamada M, Higaki J (2011) Myocardial fibrosis attenuates the effect of cibenzoline on left ventricular diastolic function in patients with hypertrophic cardiomyopathy. J Cardiovasc Pharmacol 57:207-212

19. Phelan D, Collier P, Thavendiranathan P, Popović ZB, Hanna M, Plana JC, Marwick TH, Thomas JD (2012) Relative apical sparing of longitudinal strain using two-dimensional speckletracking echocardiography is both sensitive and specific for the diagnosis of cardiac amyloidosis. Heart 98:1442-1448

20. Nagueh SF, Appleton CP, Gillebert TC, Marino PN, Oh JK, Smiseth OA, Waggoner AD, Flachskampf FA, Pellikka PA, Evangelista A (2009) Recommendations for the evaluation of left ventricular diastolic function by echocardiography. Guidelines and standards. J Am Soc Echocardiol 22:107-133

21. Miyazaki S, Daimon M, Miyazaki T, Onishi Y, Koiso Y, Nishizaki Y, Ichikawa R, Chiang SJ, Makinae H, Suzuki H, Daida H (2011) Global longitudinal strain in relation to the severity of aortic stenosis: a two-dimensional speckle-tracking study. Echocardiography 28:703-708

22. Young AA, Kramer CM, Ferrari VA, Axel L, Reichek N (1994) Three-dimensional left ventricular deformation in hypertrophic cardiomyopathy. Circulation 90:854-867

23. Van Dalen BM, Kauer F, Soliman OI, Vletter WB, Michels M, Ten Cate FJ, Geleijnse ML (2009) Influence of the pattern of hypertrophy on left ventricular twist in hypertrophic cardiomyopathy. Heart 95:657-661

24. Urbano Moral JA, Arias Godinez JA, Maron MS, Malik R, Eagan JE, Patel AR, Pandian NG (2011) Left ventricular twist mechanics in hypertrophic cardiomyopathy assessed by threedimensional speckle tracking echocardiography. Am J Cardiol 108:1788-1795

25. Serri K, Reant P, Lafitte M, Berhouet M, LeBouffos V, Roudaut R, Lafite S (2006) Global and regional myocardial function quantification by two-dimensional strain: application in hypertrophic cardiomyopathy. J Am Coll Cardiol 47:1175-1181
26. Tanaka H, Tatsumi K, Matsumoto K, Kawai H, Hirata K (2013) Emerging role of three-dimensional speckle tracking strain for accurate quantification of left ventricular dyssnchrony. Echocardiography 30:E292-E295

27. Maron BJ (2010) Contemporary insights and strategies for risk stratification and prevention of sudden death in hypertrophic cardiomyopathy. Circulation 121:445-456

28. Popovic ZB, Kwon DH, Mishra M, Buakhamsri A, Greenberg NL, Thamilarasan M, Flamm SD, Thomas JD, Lever HM, Desai MY (2008) Association between regional ventricular function and myocardial fibrosis in hypertrophic cardiomyopathy assessed by speckle tracking echocardiography and delayed hyperenhancement magnetic resonance imaging. J Am Soc Echocardiol 21:1299-1305

29. Huang J, Ni XD, Hu YP, Song ZW, Yang WY, Xu R (2011) Left ventricular longitudinal rotation changes in patients with dilated cardiomyopathy detected by two-dimensional speckle tracking imaging. Zhonghua Xin Xue Guan Bing Za Zhi 39:920-924

30. Nagakura T, Takeuchi M, Yoshitani H, Nakai H, Nishikage T, Kokumai M, Otani S, Yoshiyama M, Yoshikawa J (2007) Hypertrophic cardiomyopathy is associated with more severe left ventricular dyssychrony than is hypertensive left ventricular hypertrophy. Echocardiography 24:677-684

31. Tigen K, Sunbul M, Karaahmet T, Dundar C, Ozben B, Guler A, Cincin A, Bulut M, Sari I, Basaran Y (2013) Left ventricular and atrial functions in hypertrophic cardiomyopathy patients with very high LVOT gradient: a speckle tracking echocardiographic study. Echocardiography. doi:10.1111/echo.12482

32. Saito M, Okayama H, Yoshii T, Higashi H, Morioka H, Hiasa G, Sumimoto $\mathrm{T}$, Inaba $\mathrm{S}$, Nishimura $\mathrm{K}$, Inoue $\mathrm{K}$, Ogimoto $\mathrm{A}$, Shigematsu Y, Hamada M, Higaki J (2012) Clinical significance of global two-dimensional strain as a surrogate parameter of myocardial fibrosis and cardiac events in patients with hypertrophic cardiomyopathy. Eur Heart J Cardiovasc Imaging 13:617-623

33. Reant P, Dijos M, Donal E, Mignot A, Ritter P, Bordachar P, Dos Santos P, Leclercq C, Roudaut R, Habib G, Lafitte S (2010) Systolic time intervals as simple echocardiographic parameters of left ventricular systolic performance: correlation with ejection fraction and longitudinal two-dimensional strain. Eur J Echocardiogr 11:834-844

34. Nishikage T, Nakai H, Mor-Avi V, Lang RM, Salgo IS, Settlemier SH, Husson S, Takeuchi M (2009) Quantitative assessment of left ventricular volume and ejection fraction using twodimensional speckle tracking echocardiography. Eur J Echocardiogr 10:82-88 